Textures and Microstructures, Vol. 34, pp. 105-118 Reprints available directly from the publisher Photocopying permitted by license only
C) 2000 OPA (Overseas Publishers Association) N.V.

Published by license under the Gordon and Breach Science

Publishers imprint. Printed in Malaysia.

\title{
TEXTURE CONTROL IN THIN FILMS USING ION BOMBARDMENT
}

\author{
G.S. WAS ${ }^{\mathrm{a}, *}$, H. JI ${ }^{\mathrm{b}}$ and $\mathrm{Z} . \mathrm{MA}^{\mathrm{a}}$ \\ a Department of Nuclear Engineering and Radiological Sciences, \\ b Department of Physics, The University of Michigan, 1921 Cooley Bldg., \\ Ann Arbor, MI 48109-2104, USA
}

(Received 8 October 1999)

\begin{abstract}
The development of texture in thin films under ion bombardment is believed to occur due to the preferential growth of the aligned grains in the film relative to the unaligned grains. The difference in growth rates between aligned and unaligned grains results in the development of texture with increasing thickness. Both out-of-plane (fiber) and in-plane texture can be controlled during ion bombardment. Experiments were performed to create a (110) out-ofplane texture in thin aluminum films and to create a (110) in-plane texture in niobium films. Results showed that the texture in both cases increases in strength with depth, and that for $500 \mathrm{~nm}$ Al films, the (110) texture was stronger than the thermodynamically-preferred (111) texture obtained by physical vapor deposition. Results confirm a texturing mechanism based on ion channeling and preferential sputtering.
\end{abstract}

Keywords: IBAD; Niobium; Aluminum; Texture; Ion channeling; Sputtering

PACS: 61.66.f, 61.10.My, 68.55.a, 68.55.Jk

\section{INTRODUCTION}

Ion beam bombardment during film deposition has been found to have a pronounced effect on the establishment of texture in thin films (e.g. Harper et al., 1986; Yu et al., 1986; Sonnenberg et al., 1993; Ji et al., 1997; Rauschenbach et al., 1997). It is believed that the preferential growth of

\footnotetext{
* Corresponding author. Tel.: (734)763-4675. Fax: (734)763-4540.
}

E-mail: gsw@umich.edu. 
grains with the channeling direction aligned with the ion beam due to reduced sputtering is responsible for the established texture, e.g. Bradley et al. $(1986,1987)$. The difference in sputtering yields between aligned and unaligned grains can be as high as a factor of 5 in some materials (Roosendaal, 1981). This difference leads to a larger net growth rate for aligned grains than for unaligned grains. The newly deposited layer grows epitaxially on grains with low sputter yield orientation, and these grains will eventually dominate the film. This same process is also expected to control the surface roughness evolution, especially under deposition conditions where the surface diffusion is very limited.

The concepts of ion channeling and preferential sputtering are shown in the schematic diagram in Fig. 1. An ion impinging on a crystal in a direction aligned with the open channels in that crystal will become trapped in the channel and lose energy slowly by electronic energy transfer rather than by making high angle nuclear collisions. The net result is transport of the atom deep into the crystal and deposition of energy away from the surface. The deposition of energy deep in the crystal also means that the sputtering yield will be low for channeled ions. This is in contrast with the interaction of an ion with a crystal in an unaligned orientation. In this case, the ion has a higher probability of causing a high angle collision near the surface, resulting in energy

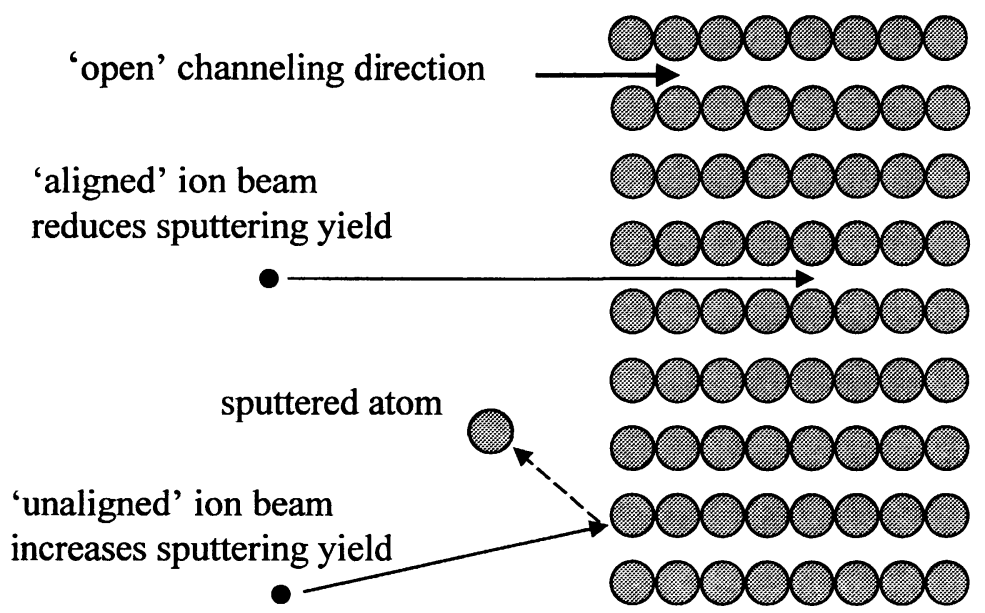

FIGURE 1 Schematic of processes of ion channeling and sputtering in a crystal whose open channels are aligned with the ion beam. 
deposition by elastic interactions near the surface and a higher sputtering yield. Hence, an observable difference between channeled and nonchanneled ions is a lower sputtering yield in the case of channeling.

This mechanism can also be applied to film growth. During deposition, crystallites are formed on the substrate with a number of different orientations. If an ion beam were directed onto the surface during film growth and the ion energy exceeded the channeling energy, the crystallites with open channels in the beam direction will have a lower sputtering yield than those with channels that are not aligned with the beam. The result is a lower sputtering rate for the aligned crystallites. Crystallites whose channeling directions are aligned with the beam will have a higher survival probability based on a reduced sputtering yield. The reduced sputtering yield also means an increased growth rate for aligned grains. This concept of texture development can be applied to control both out-of-plane and in-plane texture.

Figure 2 shows a schematic of the control of texture in an fcc film during ion beam assisted deposition (IBAD). In fcc metals, the growth plane is (111) due to its high packing density and low energy. That is, this plane is thermodynamically preferred. The easiest channeling direction in the fcc lattice is [110]. Hence, if an ion beam is directed normal to the

\section{Aluminum}

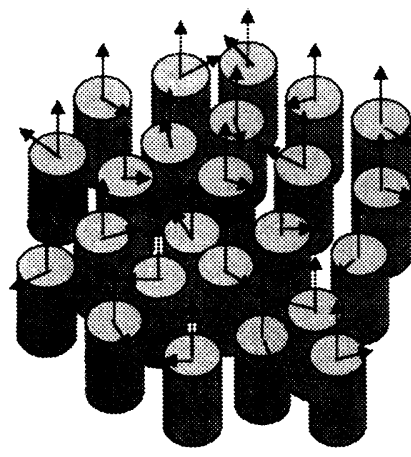

Out-of-plane (fiber) texture
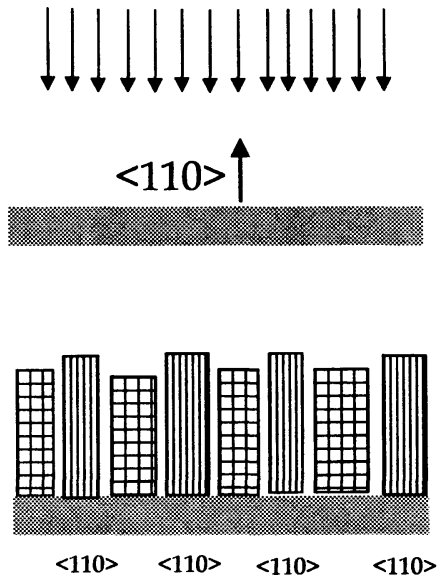

FIGURE 2 Control of out-of-plane texture in a growing fcc film by ion bombardment during deposition. 
surface, then crystallites with their [110] directions in the beam direction will be favored, and a (110) out-of-plane texture is favored. By virtue of the higher survival probability of [110]-aligned grains vs. [111]-aligned grains, the (110) texture should be preferred. Since this texture is highly non-equilibrium, low atom mobility (low film temperature) is an important factor in retaining the ion-induced (110) texture. The result is a strong (110) texture in which the grains nucleate and grow faster than the (111) grains by virtue of the lower sputtering rate and reduced surface mobility.

The control of in-plane texture occurs in much the same manner as control of out-of-plane texture, and is shown in the schematic in Fig. 3. The example we use is the growth of a bcc (e.g. Nb) film under ion bombardment. Niobium displays a (110) growth texture when deposited by physical vapor deposition. The easiest channeling direction in the bcc lattice is [100]. However, the aim here is to control the in-plane texture, not the out-of-plane texture. A schematic of the crystal orientation of $\mathrm{Nb}$ on a substrate is shown in Fig. 4, which shows how the in-plane texture is controlled. Note that the $\mathrm{Nb}$ crystal has a (110) growth texture in which the [110] direction is normal to the substrate surface. The [100] direction makes an angle of $45^{\circ}$ with [110]. Hence, ion bombardment at an angle of $45^{\circ}$ (angle $\alpha$ ) will result in the alignment of the [100] direction with the
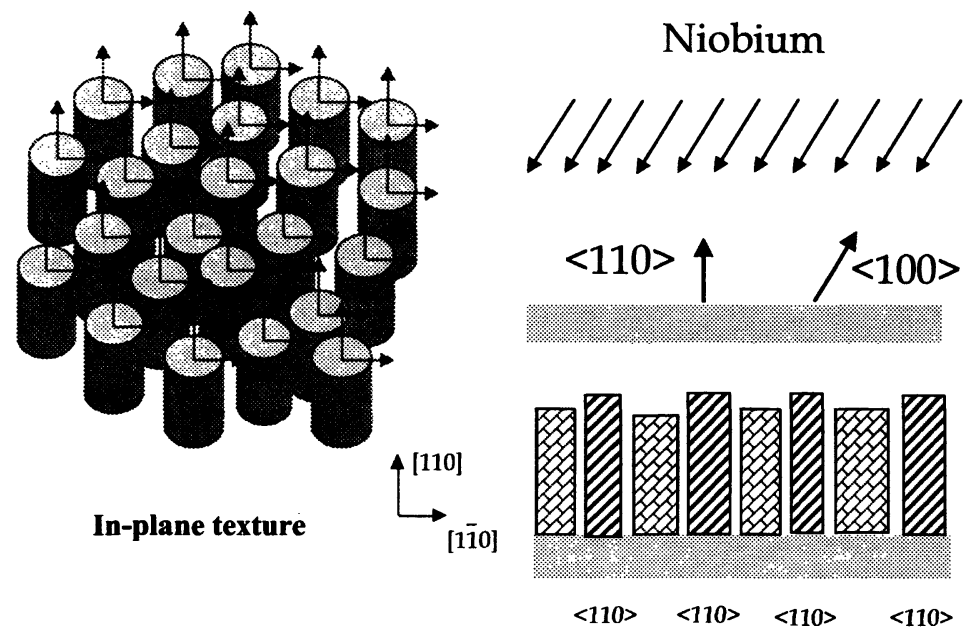

FIGURE 3 Control of in-plane texture in a growing bcc film by ion bombardment during deposition. 


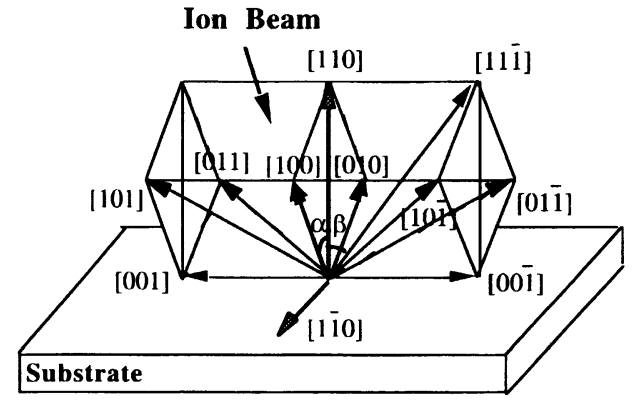

$$
\begin{aligned}
& \alpha=45^{\circ} \\
& \beta=35.26^{\circ}
\end{aligned}
$$

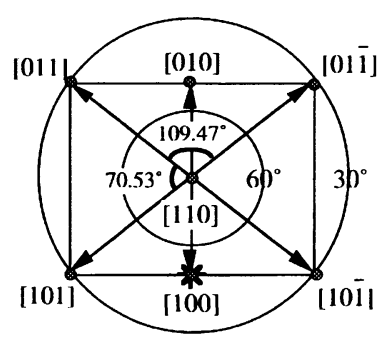

* Ion Beam Direction

FIGURE 4 Crystal structure of bcc niobium growing with a (110) out-of-plane texture.

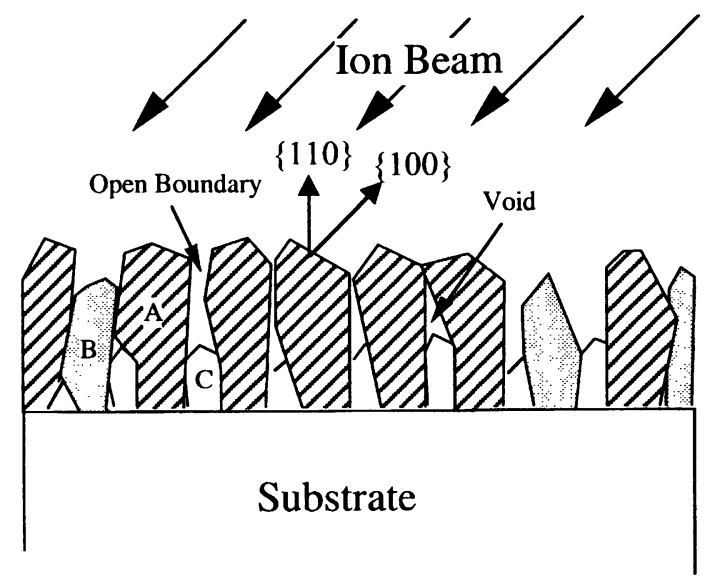

FIGURE 5 Schematic diagram of the growth and bridge-over of aligned grains in a film made using ion assisted deposition.

ion beam. This forces the [110] in-plane direction to be parallel with the projection of the ion beam on the film surface, resulting in an in-plane [110] texture. Ion bombardment does not disturb the out-of-plane texture. Thus, the film formed under an ion assist grows with a $\{110\}$ out-of-plane texture and a $\{110\}$ in-plane texture.

In both cases, subsequent film growth is governed by the differences in sputtering rate between aligned and unaligned grains. The schematic in Fig. 5 shows that as growth proceeds, aligned grains grow at a faster rate 
than do unaligned grains. The aligned grains eventually "bridge-over" and coalesce, resulting in saturation of the film texture. The difference in growth rates and the occurrence of bridge-over also explain the development of the surface roughness in textured films, e.g. Ji (1999a,b).

This paper focuses on the development of out-of-plane and in-plane textures in films produced by ion-assisted deposition. Aluminum is used to demonstrate out-of-plane texture control and $\mathrm{Nb}$ is used as an example of in-plane texture development.

\section{EXPERIMENTAL PROCEDURE}

\section{Out-of-Plane Texture Control in Aluminum}

The deposition of aluminum films was performed in an ultrahigh vacuum chamber with a base pressure of $5 \times 10^{-10}$ torr. The system consists of two $6 \mathrm{~kW}$ electron beam gun hearths and a $3 \mathrm{~cm} \mathrm{Kaufman} \mathrm{ion}$ source. The deposition rate for all experiments was maintained between 0.15 and $0.21 \mathrm{~nm} / \mathrm{s}$. A steady deposition rate is achieved by negative feedback control of the e-gun power using the real time rate signals from the quartz crystal thickness monitors, and the ion beam flux is measured by a Faraday cup.

Except for a PVD sample which was used for comparison in each set of depositions, all films were bombarded with a beam of $\mathrm{Ne}^{+}$ions with energy between 200 and $1200 \mathrm{eV}$. The ion current density was between 43 and $193 \mu \mathrm{A} / \mathrm{cm}^{2}$, yielding an ion-to-atom arrival rate ratio $(R)$ of 0.3 to 1.0 and normalized energies between 120 and $1200 \mathrm{eV} /$ atom. Films were deposited onto (100) silicon wafers of thickness $0.5 \mathrm{~mm}$. Samples were mounted to a copper block and cooled from the back with either liquid nitrogen $\left(\mathrm{LN}_{2}\right)$ or water or were deposited without cooling. All depositions were made with the substrate normal to the ion flux and at $45^{\circ}$ to the vapor flux as shown in Fig. 6 . The target thickness for all films was $500 \mathrm{~nm}$. For films made with an ion beam assist, sputtering of the substrate by energetic ions was compensated by increasing the deposition time. The resulting film thickness ranged from 420 to $550 \mathrm{~nm}$, as measured by a Dektak 3 profilometer.

Texture was characterized using Schultz's X-ray pole figure method and from $\theta-2 \theta \mathrm{X}$-ray diffraction scans about a $0^{\circ}$ tilt angle, using a $\mathrm{Cu} \mathrm{K}_{\alpha}$ $\mathrm{X}$-ray source at $40 \mathrm{kV}$ and a current of $110 \mathrm{~mA}$. For each film the areas 


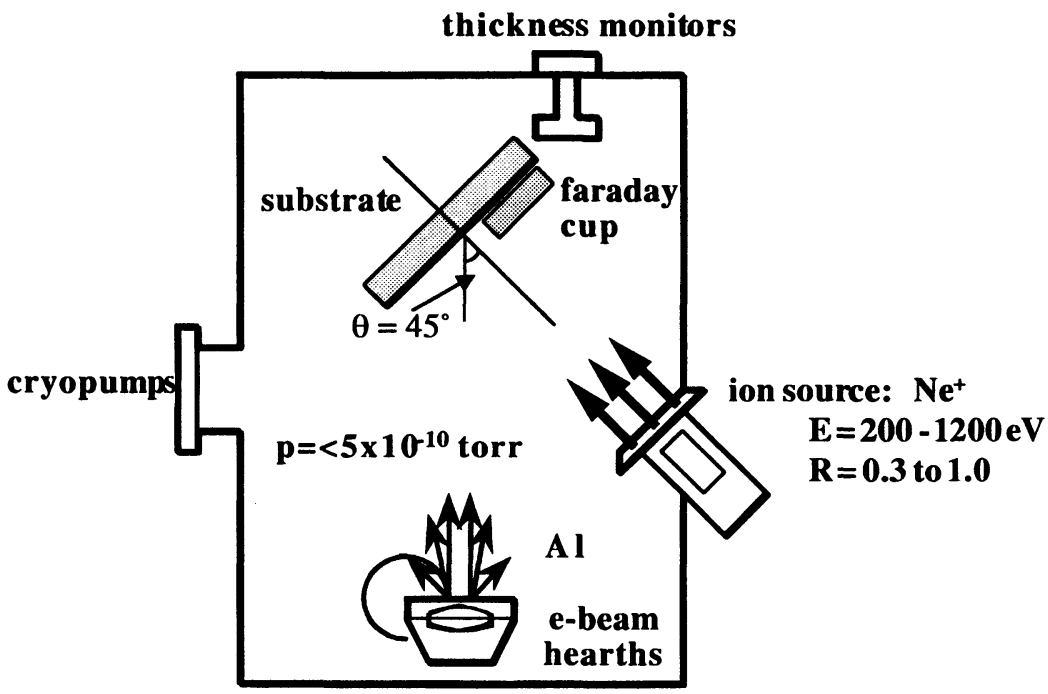

FIGURE 6 Experimental arrangement for out-of-plane texture control in aluminum.

under the (220) and (111) peaks were measured after background subtraction. The strength of the fiber texture was determined by plotting the ratio of the areas of the (220) and (111) peaks, $I_{(220)} / I_{(111)}$.

\section{In-Plane Texture Control in Niobium}

Niobium films were synthesized in the same chamber as were aluminum films. Depositions were made onto (100) silicon, and the deposition rate for all experiments was kept close to $0.5 \mathrm{~nm} / \mathrm{s}$. Film thickness ranged from 20 to $940 \mathrm{~nm}$. Except for one sample which was deposited without ion bombardment (PVD), all samples were bombarded with an $\mathrm{Ar}^{+}$ion beam at an energy of $1000 \mathrm{eV}$ and an ion flux of $193.0 \mathrm{~mA} / \mathrm{cm}^{2}(R=0.4)$. The ion beam incident angle was $50^{\circ}$ from the substrate surface normal (Fig. 7). The actual film thickness is about $71 \%$ of the nominal film thickness (film thickness without taking into account the effect of sputtering) under this deposition condition. All depositions were performed at ambient substrate temperature.

The in-plane texture in niobium films was measured by the pole figure technique using the Schultz reflection method. Two pole figures, for 


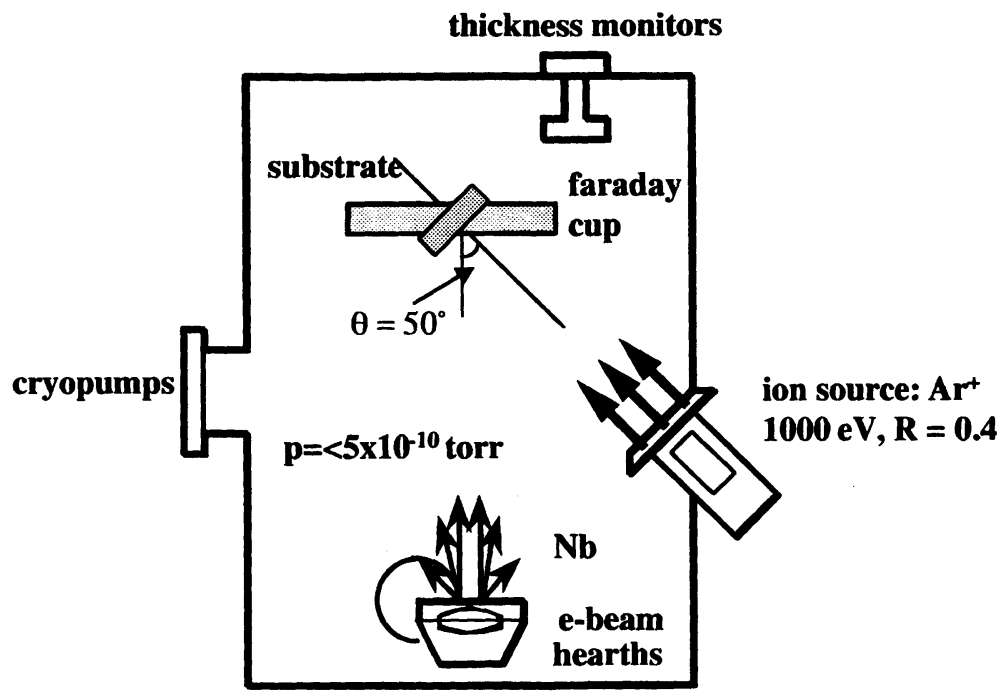

FIGURE 7 Experimental arrangement for in-plane texture control in niobium.

$\{110\}$ and $\{200\}$ planes, were measured on these niobium films. The intensities of the $\{110\}$ and $\{200\}$ Bragg diffraction peaks were measured at Bragg angles $2 \theta=38.2^{\circ}$ and $55.1^{\circ}$, respectively.

In-plane texture in niobium films was also studied using cross-section TEM. Samples were prepared with an ultramicrotome using a technique developed by Schubert-Bischoff(1997). Soda-lime-silica glass substrate was used in this study. A dark field image from the (110) diffraction of the cross-section of the niobium film was obtained, and the average brightness at a certain film depth was related to the degree of grain orientation alignment in the film at that depth.

\section{RESULTS AND DISCUSSION}

\section{Out-of-Plane Texture Control in Aluminum}

Results show that on $\mathrm{Si}(100)$, increasing the normalized energy results in an increase in $I_{(220)}$ relative to $I_{(111)}$. The dependence of the (220) and (111) X-ray peaks on the normalized energy for the case of Si (100) wafers between $\mathrm{LN}_{2}$ temperature and room temperature is shown in Fig. 8. Note that the (220) peak becomes progressively stronger relative to the (111) peak with increasing normalized energy. The dashed line 


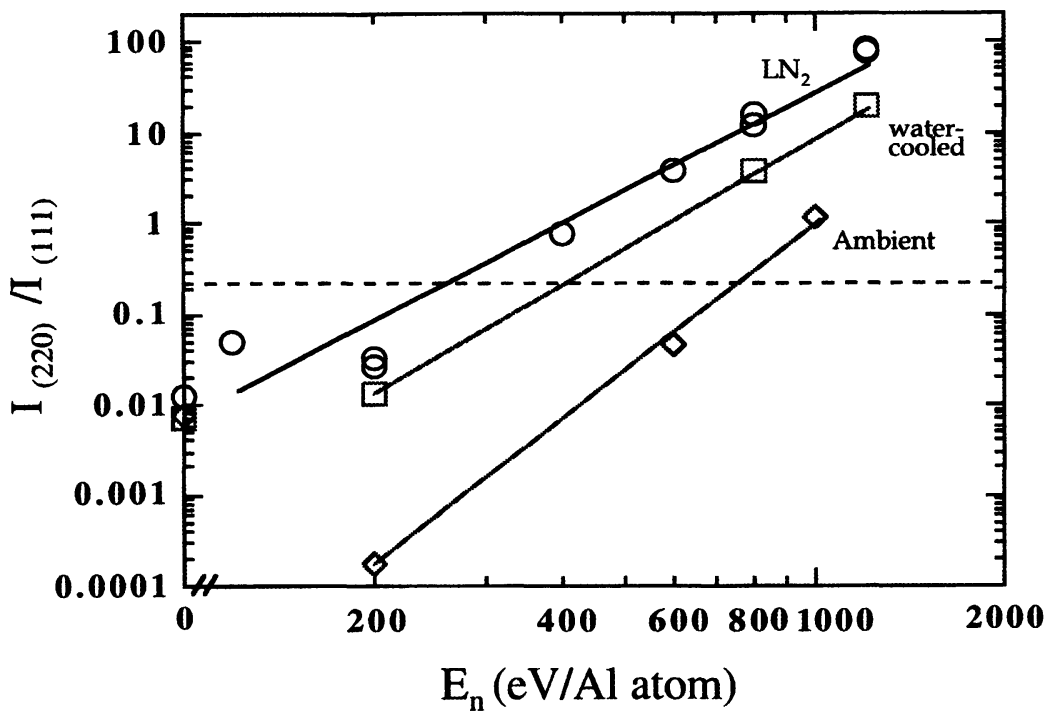

FIGURE 8 Intensity ratio of (220)/(111) X-ray peaks in $500 \mathrm{~nm} \mathrm{Al} \mathrm{films} \mathrm{made} \mathrm{by}$ ion beam assisted deposition using $\mathrm{Ne}^{+}$ions.

is the random texture ratio plotted for reference. Since the sputter yield is proportional to both ion energy (in this energy range) and ion flux, it is expected that the sputter yield difference between (110)- and (111)oriented grains will increase with normalized ion energy (Was et al., 1997). The formation of a (110) fiber texture is favored by a low deposition temperature because the adatom diffusion which assists the thermodynamically-preferred (111) texture is suppressed, allowing ion channeling and preferential sputtering to control the growth of (110)-oriented grains. Hence, depositions at lower temperature result in a stronger (110) texture.

Figure 8 also shows that for the ambient temperature depositions at the lowest normalized energy $(200 \mathrm{eV} /$ atom) the (111) fiber texture was actually stronger than in the PVD films without ion bombardment. This result is consistent with the work of Masaki et al. (1991). At temperatures where adatom diffusion is high relative to the deposition rate, a small increase in adatom diffusion produced by the ion beam assists the formation of the thermodynamically-preferred texture. Experiments conducted on glass shows similar results, indicating that the substrate does not control texture nucleation or development (Ma and Was, 1998). 


\section{In-Plane Texture Control in Niobium}

Figure 9 shows the $\{110\}$ and $\{200\}$ pole figures for niobium films deposited on (100) silicon substrates under two different conditions: PVD (Fig. 9(a)), and IBAD at $E=1000 \mathrm{eV}, R=0.4$ (Fig. 9(b)). It should be noted that the ion beam incident direction was at a tilt angle of $50^{\circ}$ from the substrate surface normal and an azimuthal angle of $0^{\circ}$. Both PVD and IBAD samples have intensity maxima at the center of the $\{110\}$ pole figures. Additional maxima appear on the $30^{\circ}$ ring as indicated in Fig. 9. For the PVD sample, the intensity on this $30^{\circ}$ ring was azimuthally uniform, indicating an absence of in-plane texture. The IBAD sample had four distinct intensity maxima at preferred azimuthal directions on the $30^{\circ}$ ring of the $\{110\}$ pole figure, reflecting a strong $\{110\}$ in-plane

(a)

$\{110\}$

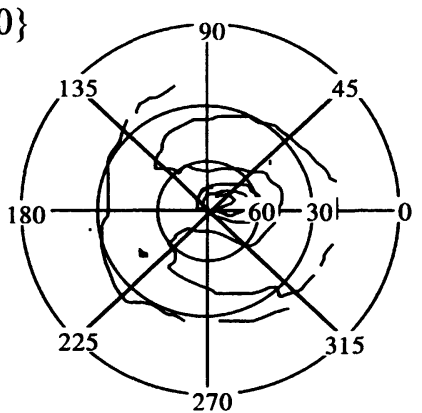

$\{110\}$

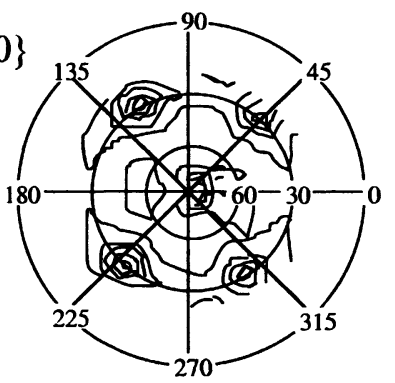

$\{200\}$

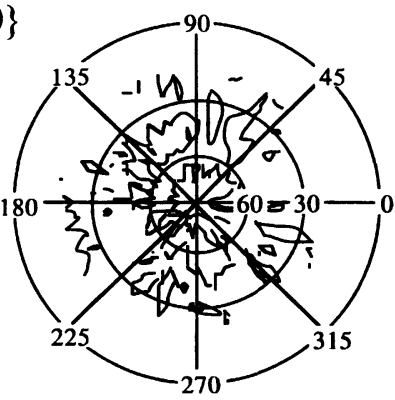

$\{200\}$

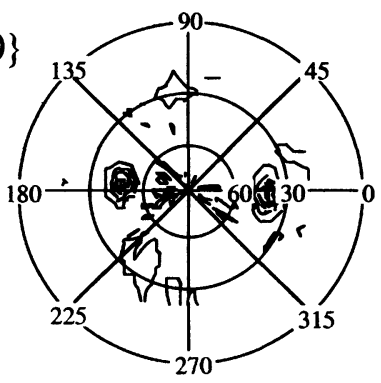

(b)

$$
\begin{aligned}
& \text { degree of texture } \\
& \text { (intensity ratio) }
\end{aligned}=\frac{\mathrm{I}_{\mathrm{avg}}^{30^{\circ}}}{\mathrm{I}^{0^{\circ}}}
$$

FIGURE 9 (110) and (200) pole figures of niobium films made by (a) PVD and (b) IBAD (1000 eV, $R=0.4$ ) deposited onto (100) silicon substrates. 
texture. The azimuthal angles of these four intensity maxima are $47^{\circ}$, $117.5^{\circ}, 227^{\circ}$, and $297.5^{\circ}$, respectively, and are separated by angles, of $70.5^{\circ}$ and $109.5^{\circ}$.

The $\{200\}$ pole figure of the PVD sample has an intensity maximum on the $45^{\circ}$ ring. In the IBAD film, two intensity maxima, identified as $\{200\}$ poles, occur on the $45^{\circ}$ ring at azimuthal angles of $-7.5^{\circ}$ and $172.5^{\circ}$. These two maxima also bisect the two larger angles $\left(109.5^{\circ}\right)$ between the $\{110\}$ poles in the $\{110\}$ pole figure. The results for an IBAD niobium film deposited on amorphous glass substrate are the same as for the (100) $\mathrm{Si}$ substrate $(\mathrm{Ji}, 1998 \mathrm{~b}$ ) indicating that the substrate effect (epitaxial growth) cannot be responsible for the in-plane texture in the films. The development of $\{110\}$ in-plane texture in the IBAD films was induced by ion beam bombardment.

The degree of $\{110\}$ in-plane texture was quantified using the parameter $D$ (intensity ratio) defined as

$$
D=I_{\text {avg }}^{30^{\circ}} / I^{0^{\circ}},
$$

where $I_{\text {avg }}^{30^{\circ}}$ is the average intensity of the four $\{110\}$ poles on the $30^{\circ}$ ring and $I^{\circ}$ is the intensity of the center $\{110\}$ pole (Ji et al., 1997). As shown in Fig. 10, the degree of in-plane texture increased as the film grew

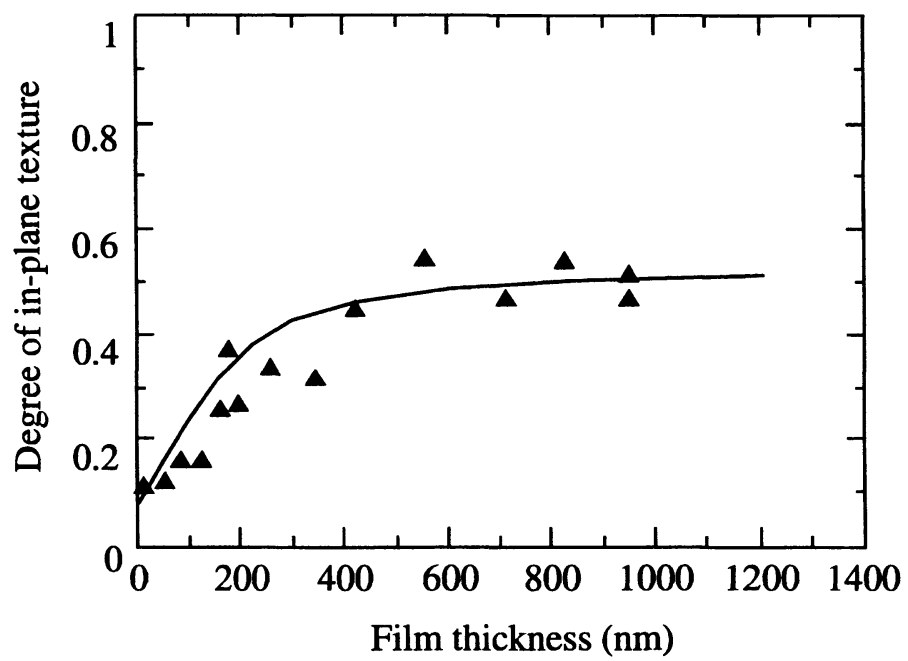

FIGURE 10 Degree of in-plane texture vs. film thickness for IBAD $(1000 \mathrm{eV}$, $R=0.4)$ niobium films deposited onto (100) silicon substrates. 
thicker until the degree of in-plane texture appears to saturate. However, the texture measured by the pole figure technique is integrated or "averaged" over the entire film thickness, and thus, it does not provide information on texture at a specific film depth. Thus, cross-section TEM was employed to measure the texture at various film depths.

As shown in Fig. 11, the dark-field image of a selected portion of the (110) diffraction ring was obtained. In this image, the largest selected area aperture was used so the entire film thickness of the film was included. The average brightness of the image at different film depth (or distance from the film/substrate interface), which is indicative of the number of grains contributing to the (110) diffraction at this specific spatial direction, is also plotted along with the image. The average brightness increased with the film depth and then leveled off at about $250 \mathrm{~nm}$. The correlation between the saturation film thickness for local
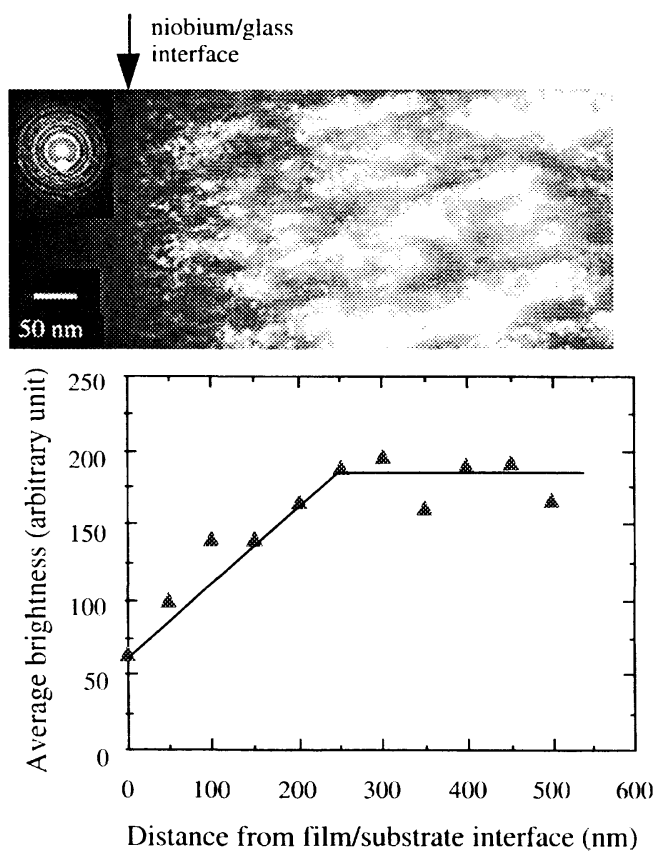

FIGURE 11 Cross-section TEM micrograph of the dark field image from the (110) diffraction of an IBAD $\mathrm{Nb}$ film $(1000 \mathrm{eV}, R=0.4)$. The graph plots the average image brightness (degree of grain orientation alignment) as a function of the distance from the film/substrate interface. 
grain alignment $(250 \mathrm{~nm})$ and integrated in-plane texture $(570 \mathrm{~nm})$ is good and is discussed in more detail in Ji (1998b). Nevertheless, both sets of data substantiate the development of a preferred (110) in-plane texture with increasing film thickness.

\section{SUMMARY}

Aluminum and niobium films were deposited onto (100) Si substrates using an ion beam assist to control the crystallographic texture. Aluminum films were bombarded with $\mathrm{Ne}^{+}$ions during deposition in a direction normal to the substrate, resulting in the development of a (110) out-of-plane texture. The strength of the texture increased with normalized energy and was stabilized by lower deposition temperatures. Niobium films were bombarded with $\mathrm{Ar}^{+}$ions during deposition at an angle of $50^{\circ}$ with respect to the substrate to control the in-plane texture. The films displayed a (110) in-plane texture induced by the ion beam and a (110) out-of-plane texture that was unaffected by the ion beam. The strength of the texture increased with thickness of the deposited film. Both results support a texturing mechanism based on ion channeling and preferred sputtering of aligned grains during the growth of the deposited film.

\section{Acknowledgments}

The authors would like to acknowledge the Michigan Ion Beam Laboratory for Surface Modification and Analysis and the Electron Microbeam Analysis Laboratory at the University of Michigan for the use of their facilities. This work is supported under NSF grant \#DMR9411141 and under the Air Force Office of Scientific Research grant \#F49620-95-1-0524.

\section{References}

Bradley, R.M., Harper, J.M.E. and Smith, D.A. (1986). Theory of thin-film orientation by ion bombardment during deposition. J. Appl. Phys., 60(12), 4160-4164.

Bradley, R.M., Harper, J.M.E. and Smith, D.A. (1987). Theory of thin-film orientation by ion bombardment during deposition. J. Vac. Sci. Technol., A5(4), 1792-1793. 
Harper, J.M.E., Smith, D.A., Yu, L.S. and Cuomo, J.J. (1986). Microstructure of niobium films oriented by non-normal incidence ion bombardment during growth. In BeamSolid Interactions and Phase transformations, Vol. 51, edited by H. Kurz, G.L. Olson and J.M. Poate, pp. 343-348. Pittsburgh: Mater. Res. Soc.

Ji, H. and Was, G.S. (1999a). Linkage between crystallographic texture and surface roughness in niobium films synthesized by ion beam assisted deposition. Nucl. Instr. Meth. in Phys. Res., B148, 880-885.

$\mathrm{Ji}, \mathrm{H}$. and Was, G.S. (1999b). Mechanism of in-plane texture development by ion beam assisted deposition. Submitted to J. Mater. Res., 14(6), 2524-2532.

Ji, H., Was, G.S., Jones, J.W. and Moody, N.R. (1997). Effect of ion bombardment on in-plane texture, surface morphology and microstructure of vapor deposited $\mathrm{Nb}$ thin films. J. Appl. Phys., 81(10), 6754-6767.

Ma, Z. and Was, G.S. (1999). Aluminum metallization for flat panel displays using ion beam assisted physical vapor deposition. Submitted to J. Mater. Res., 14(10), 40514061.

Masaki, S., Kobayashi, H. and Morisaki, H. (1991). Ion-beam-assisted deposition of Al films with strong preferential texture. Nucl. Instr. Meth., B59/60, 292-296.

Rauschenbach, B., Zeitler, M., Gerlach, J.W. and Stritzker, B. (1997). Manipulation of texture by low-energy ion beams: example ion assisted deposition of titanium nitride. Nucl. Instr. Meth. in Phys. Res. B, 127/128, 813-816.

Roosendaal, H.E. (1981). Sputtering by particle bombardment I - Physical sputtering of single-element solids. In Topics in Applied Physics, Vol. 47, edited by R. Behrisch, pp. 219-256. Berlin: Springer.

Schubert-Bischoff, P. and Krist, T. (1997). Fast cross-sectioning technique for thin films by ultramicrotomy. In Microscopy and Microanalysis 1997, edited by G.W. Bailey, R.V.W. Dimlich, K.B. Alexander, J.J. McCarthy and T.P. Pretlow, pp. 359-360. New York: Springer-Verlag.

Sonnenberg, N., Longo, A.S., Cima, M.J., Chang, B.P., Ressler, K.G., McIntyre, P.C. and Liu, Y.P. (1993). Preparation of biaxially aligned cubic zirconia films on pyrex glass substrates using ion beam assisted deposition. J. Appl. Phys., 74(2), 1027-1034.

Was, G.S., Srolovitz, D.J., Ma, Z. and Dong, L. (1997). Microstructure control for thin film metallization. In Thin-Films - Structure and Morphology, Vol. 442, edited by S.C. Moss, D. Ila, R.C. Cammarata, E.H. Chason, T.L. Einstein and E.D. Williams, pp. 311-322. Pittsburgh: Mater. Res. Soc.

Yu, L.S., Harper, J.M.E., Cuomo, J.J. and Smith, D.A. (1986). Control of thin film orientation by glancing angle ion bombardment during growth. J. Vac. Sci. Technol., A4(3), 443-447. 